\title{
Does Expectation of Correlation Breakdown in Financial Market Fulfill Itself?
}

\author{
Paolo Falbo ${ }^{1}$ and Rosanna Grassi ${ }^{2}$ \\ ${ }^{1}$ Department of Quantitative Methods, University of Brescia, 25121 Brescia, Italy \\ ${ }^{2}$ Department of Statistics and Quantitative Methods, University of Milano-Bicocca, 20126 Milano, Italy \\ Correspondence should be addressed to Paolo Falbo; paolo.falbo@unibs.it
}

Received 10 November 2014; Revised 14 July 2015; Accepted 15 July 2015

Academic Editor: Cengiz Çinar

Copyright (c) 2015 P. Falbo and R. Grassi. This is an open access article distributed under the Creative Commons Attribution License, which permits unrestricted use, distribution, and reproduction in any medium, provided the original work is properly cited.

\begin{abstract}
This paper develops a model appeared in the literature whose focus was the way rational risk averse investors anticipate the correlation breakdowns of asset returns in periods of excess demand. That model analysed the dynamics of the "expected" returns of the risky asset, and their consistency with empirical evidence. However, the same model did not provide any evidence on actual correlation generated by the dynamics of returns. A model to link asset returns to excess demand is required to analyse the implied correlation between the securities traded. In this work we estimate such a model. Results confirm that the expected and ex-post correlation tend to move closely. In other words a self-fulfilling prophecy about correlation breakdown can take place, even when rational agents dominate the financial market.
\end{abstract}

\section{Introduction}

Several studies in the literature document the so-called correlation breakdown phenomenon (also reported as run to unity) that is a sudden convergence of the correlation between the returns of traded assets to the value of 1 . In particular this effect appears during periods of financial crisis, but there is also evidence during market euphoria. In Bertero and Mayer [1] and King and Wadhwani [2] authors show an increase in the correlation of stock returns at the time of the 1987 crash. Calvo and Reinhart [3] give evidence of correlation change associated with the Mexican crisis, and Baig and Goldfajn [4] find an increase of correlation in several East-Asian markets and currencies during the East-Asian crisis. Also Longin [5], Hartmann et al. [6], and Bae et al. [7] propose models based on extreme value theory, whereas Ramchand and Susmel [8], Ang and Bekaert [9], and Chesney and Jondeau [10] analyze Markov switching models. Loretan and English [11] identify in the "breakdowns of historical correlations" the origin of the Russian default in August 1998 and Karolyi and Stulz [12] show the existence of factors influencing joint movements in the US-Japan markets using regression methods. Situations, where the "historical" correlation among the assets traded on the same and even on different markets breaks down and asset suddenly shows a "run to unity" of this correlation, represent clearly a major problem for investors, since the risk protection usually guaranteed by the diversification of their portfolios is lost; besides these events tend to show exactly in the moment of major need.

The study of financial contagion was developed mostly around the notion of correlation breakdown (see $[1-3,13,14]$ ), so this paradigm helps to explain important dynamics of financial markets such as financial crises and speculative bubbles.

In this work we analyze the model of Falbo and Grassi [15] where the price dynamics of two assets (a high-risk and a low-risk ones) is subject to a time varying correlation. In particular, the authors consider the case where rational investors use expectation of excess demand to forecast the correlation between the assets to compose their investment portfolio. Their model explains several market dynamics, including market crashes, creation of rational bubbles, or cycles of diverse periods. Such results depend on the initial conditions as well as the percentage composition of the 
market between rational and irrational agents and their attitude to respond more or less aggressively to shocks in the excess demand. At the heart of their model there is the hypothesis that rational agents update the estimates of variance and covariance functions, depending on the excess demand of the risky asset.

Despite its interesting results, that model is missing an important component. In particular it does not include an equation on the returns of the low-risky asset and therefore it cannot provide clear insights into the origin of the time varying correlation. Besides, the absence of a conclusive evidence that the model does generate time varying correlation as a function of the excess demand of the risky asset weakens the hypothesis that rational agents are correct in linking their estimation of the correlation to the excess demand.

The main objective of this paper is therefore to complete the analysis of the model of Falbo and Grassi. To this purpose, we introduce an equation where the returns of the lowrisk asset depend on the excess demand of the risky asset. This model is inspired by the observation of empirical data. This is relevant because next to supporting the theoretical consistency of the entire model such equation gives it also an empirical support. It is worth pointing out that, to the best of our knowledge, the literature on time varying correlation and correlation breakdown is entirely based on empirical and econometric analysis, so this work is a first attempt to provide a theoretical framework to explain and model the origin of this relevant feature of financial markets.

This work is organized as follows. Section 2 summarizes the main equations of the original model, with the purpose of making this paper self-contained. In Section 3 the equation for the low-risk asset is introduced and its empirical evidence is discussed. Section 4 develops the transition dynamics of the model integrated with the new equation of the low-risk assets. The results are used to discuss the consistency of the model with its central hypothesis on how rational agents estimate the correlation between the traded assets. Section 5 concludes.

\section{A Contagion Model with Rational and Speculative Investors}

Moving from the settings in Falbo and Grassi [15] that we partially recover here to introduce the notation and to make this paper minimally self-contained, we consider a market with two types of agents, rational investors and speculators. These agents interact in a discrete time framework by trading two kinds of assets, a stock $s$ and a consol bond $b$, with different risk levels and different expected returns, $r_{s}$ and $r_{b}$, respectively.

Both types of investors look at excess demand, but with different premises.

Speculators are not informed of the true value of the risky asset at time $t$ and they place their investing decision on the basis of the excess demand of the previous period. In particular they will introduce an excess demand for the risky asset based on the formula $w_{t}^{S}=\chi_{1}\left(w_{t-1} /\left(1+\left|w_{t-1}\right|\right)\right)$.
Parameter $\chi_{1}>0$ or $\chi_{1}<0$ describes, respectively, momentum or contrarian strategies.

Rational investors behave differently: at the beginning of each period $t$, they update their estimate of the expected return $r_{s}$ of the high-risk asset comparing its current price $P_{t}$ with its true value $\bar{P}$ (this knowledge is their information advantage):

$$
r_{s, t}=\ln \left(\frac{P_{t}+k\left(\bar{P}-P_{t}\right)}{P_{t}}\right) .
$$

Observe that this formula describes a mean reverting dynamic of the expected return, where $k \in(0,1)$ is a coefficient of reversion speed. In general, the expected return differs from the actual one, since it depends on the excess demand:

$$
\Delta p_{t}=\ln \frac{P_{t}}{P_{t-1}}=r_{s, t-1}+\lambda w_{t-1} .
$$

Rational agents diversify their investment through a Markowitz portfolio model estimating the correlation between the two assets depending on the excess demand. In particular they believe that during market phases with high or low excess demand, correlation between the assets tends to unity. So excess demand influences their portfolio decision as we will discuss later on.

A feedback develops at this point, since rational agents also influence the excess demand. The equation modeling the excess demand generated by the rational agents is $w_{t}^{R}=$ $\chi_{2}\left(q_{t}^{*}\left(w_{t-1}, r_{s, t-1}\right)-q_{t}^{*}\left(0, r_{s, t-1}\right)\right)$, where $q_{t}^{*}\left(w_{t-1}, r_{s, t-1}\right)$ is the optimal quantity of the stock in the Markowitz portfolio. Rational excess demand is a (linear) function of the difference between $q_{t}^{*}\left(w_{t-1}, r_{s, t-1}\right)$ and $q_{t}^{*}\left(0, r_{s, t-1}\right)$, modulated by a sensitivity parameter $\chi_{2} \in \mathbb{R}-\{0\}$, the share of speculators in the market.

The total market excess demand is then a convex combination of those generated by the two kinds of agents, $w_{t}=$ $Y w_{t}^{S}+(1-Y) w_{t}^{R}$, with $Y \in[0,1]$ being the share of speculators in the market.

The two-dimensional system is then the following:

$$
\begin{aligned}
w_{t}= & Y \chi_{1} \frac{w_{t-1}}{1+\left|w_{t-1}\right|} \\
& +(1-Y) \chi_{2}\left(q_{t}^{*}\left(w_{t-1}, r_{s, t-1}\right)-q_{t}^{*}\left(0, r_{s, t-1}\right)\right) \\
r_{s, t}= & \ln \left(\frac{\exp r_{s, t-1}+k-1}{\exp \left(r_{s, t-1}+\lambda w_{t-1}\right)}-(k-1)\right)
\end{aligned}
$$

which fully describes the evolution of the fundamental variables of this market, $w$ and $r_{s}$. The optimal quantity $q_{t}^{*}$ in the first equation of (3) is calculated by informed investors solving a portfolio optimization problem. In the Appendix, the expression of the solution is reported.

In such solution the central assumption consists in letting rational agents estimate the variance and correlation matrix $V_{t-1}$ of the two assets through the following equations:

$$
\begin{aligned}
& v_{t}=\alpha^{2} e^{2 s w_{t}^{2}}, \\
& \rho_{t}=-e^{-\mu w_{t}^{2}}+1 .
\end{aligned}
$$


These equations tell us that rational agents update, respectively, their estimate of the variance and correlation, observing how the market is overbuying or overselling. In particular when the current excess demand $w_{t}$ gets very large, they expect that a correlation breakdown is going to develop $\left(\rho_{t} \rightarrow 1\right.$ as $w_{t} \rightarrow \pm \infty$ for $\left.\mu>0\right)$. As a consequence of the theory of Markowitz, the portfolio of rational agents will be largely impacted in this case. The response of rational agents when they fear that the correlation gets close to 1 has in turn a large impact on $w_{t+1}$. In this way no obvious market dynamics originates, motivating the present study.

Of course, other functional forms for $\rho$ can be assumed. The key feature to be saved, to keep the economic structure of this model, is that $\rho_{t} \rightarrow 1$ as $w_{t} \rightarrow \pm \infty$. The parameter $s$ in the variance equation (4) can be either positive or negative; assuming that $s$ is positive amounts to estimating (on the side of the rational agents) that $v_{t} \rightarrow+\infty$ as $w_{t} \rightarrow \pm \infty$, whereas $v_{t} \rightarrow 0$ as $w_{t} \rightarrow \pm \infty$ for negative $s$. This is a slight modification of the original model of Falbo and Grassi [15] which generalizes possible empirical applications.

It is worth observing that the hypotheses in (4) and (5) are just concerned with estimation of rational investors. They are well distinguished from speculators, since they do not use the excess demand to work out new estimates for the expected returns.

The model of Falbo and Grassi [15] generates returns of the stock price distributed in a way consistent with the variance function in (4). However, with respect to the correlation, that paper did not say anything conclusive since the model was missing an equation linking the low-risk asset returns to the excess demand $w_{t}$. It is a major purpose of this paper to bridge this gap. By introducing an equation for the lowrisk asset, we study under which conditions the model is able to generate a correlation between the two assets and check the internal consistency of the hypothesis in (5). Proving this consistency is of primary theoretical relevance. If it is indeed confirmed, the model would supply a robust explanation of the phenomenon of the correlation breakdowns, which, to the best of our knowledge, is still missing in the literature. In particular the model explains the origin of the correlation breakdown attributing it to two (possibly concurrent) causes: the herding behavior of momentum speculators and the procyclical attempt of rational investors to protect against the correlation breakdown.

The modeling contribution of this paper consists of including in the study an equation describing the price dynamics of the low-risk asset $\left(\widehat{r}_{b, t}\right)$. Through this modification the model driving the market becomes a threedimension system:

$$
\begin{aligned}
w_{t}= & Y \chi_{1} \frac{w_{t-1}}{1+\left|w_{t-1}\right|} \\
& +(1-Y) \chi_{2}\left(q_{t}^{*}\left(w_{t-1}, r_{s, t-1}\right)-q_{t}^{*}\left(0, r_{s, t-1}\right)\right), \\
r_{s, t}= & \ln \left(\frac{\exp r_{s, t-1}+k-1}{\exp \left(r_{s, t-1}+\lambda w_{t-1}\right)}-(k-1)\right), \\
\widehat{r}_{b, t}= & a w_{t-1}^{3}-b w_{t-1}+r_{b},
\end{aligned}
$$

where $a$ and $b$ are suitable parameters. Let us observe that the new equation implies that in the absence of excess demand the realized returns of the low-risk assets coincide with their expected value. Moreover, adding such equation to system (3) does not introduce a feedback and so it does not alter the theoretical properties of that model, which can be still studied as a two-dimensional system.

\section{An Empirical Model for the Low-Risk Asset}

In their empirical application Falbo and Grassi [15] develop a proxy of the excess demand of a stock index. We recover the same equations, since that variable is also needed here to model the returns of low-risky assets. Of course excess demand is not a directly observable variable. Besides, the definition itself of excess demand is not precise, given that it would require a clear notion of what "regular" or "normal level of" demand is, which is not at all obvious. Nevertheless the concept of excess demand in the financial markets is familiar and it is often used, both in the financial literature and in the comments of specialized magazines, as an explanation of large price movements, when markets take a clear direction either increasing or decreasing.

The proxy for the excess demand for a stock index can be obtained counting the number of times that the daily returns take a plus or minus sign over a given period in the stock market. The reason why the prevalence of a sign in the returns (in a given period of time) can be considered as a proxy for the excess demand is related to contagion arguments. Indeed it can be supposed that the longer a given sign prevails on the stock market, the higher the probability that agents are sharing a common sentiment in the same period. The following two expressions count the number of times that positive and negative returns are observed from $t$ back to $t-6$ :

$$
\begin{aligned}
& \#_{t}^{+}=\sum_{i=0}^{6} I_{R^{+}}\left(\widetilde{r}_{s, t-i}\right), \\
& \#_{t}^{-}=\sum_{i=0}^{6} I_{R^{-}}\left(\widetilde{r}_{s, t-i}\right),
\end{aligned}
$$

where $I(\cdot)$ is the indicator function and $\widetilde{r}_{s, t}$ is the return observed on a stock market index at time $t$. These expressions are actually slightly modified with respect to the original proposal in [15], extending the summations to 6 instead of 5 to capture more extreme cases. The authors argue that if, at any time $t$, the counter of either sign exceeds the other, the market is expressing a "consensus" that can be taken as a measure of excess demand. In particular this consensus variable is defined as

$$
c_{t}= \begin{cases}\#_{t}^{+} & \text {if } \#_{t}^{+}>\#_{t}^{-} \\ -\#_{t}^{-} & \text {if } \#_{t}^{+}<\#_{t}^{-} \\ 0 & \text { if } \#_{t}^{+}=\#_{t}^{-}\end{cases}
$$

This variable takes values in normal conditions (i.e., no stop of the market in the period $t-6$ to $t$ ) in 


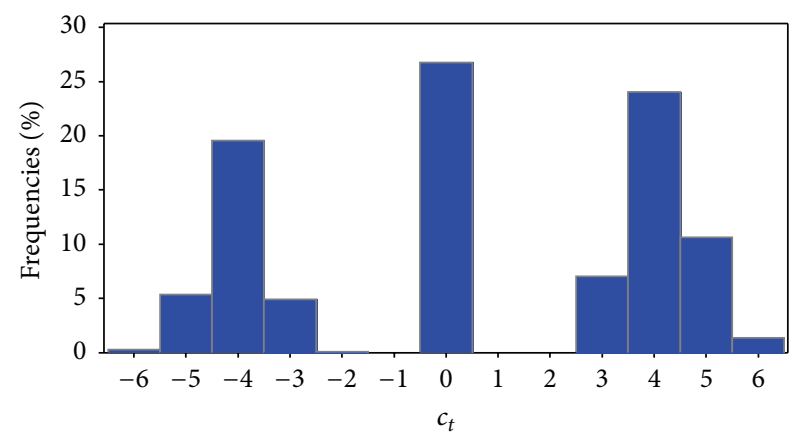

FIGURE 1: Frequency distribution of consensus variable $c_{t}$ for the US stock index (Datastream) calculated in the period of Janunary 1, 1993, to March 31, 2008.

the range $\{-6,-5,-4,0,4,5,6\}$. Usually $c_{t}$ does not take the values $\{-3,-2,-1,1,2,3\}$, given that in the period $t-6$ to $t$ such values would be exceeded by their opposite counter (e.g., supposing that, from $t-6$ to $t, 4$ negative and 3 positive returns have been observed, we would obtain $\#_{t}^{+}=3, \#_{t}^{-}=4$, and then $\left.c_{t}=-4\right)$. However it is occasionally possible that, apart from the Saturdays and Sundays (that are preliminarily excluded from the analysis), the market stops for celebrations or some other reasons. For example, it can occasionally happen that the market works only 5 days between $t-6$ and $t$. Then a possible result could be $\#_{t}^{+}=3, \#_{t}^{-}=2$, and then $c_{t}=3$.

3.1. Data. We observe the daily series of a US stock index calculated by Datastream (which is adjusted for various stock splits, mergers, etc., of individual companies) and the daily series of prices of the US Treasury bond, 30 years' maturity, issued on September 1986. The period of observation ranges from January 1, 1993, to March 31, 2008. The following graph in Figure 1 shows the frequency distribution of the proxy of the excess demand as in (8). It can be observed that the cases where $c_{t}$ is equal to -6 or 6 are rare but not negligible.

Figure 2 shows the relation between the average daily return of low-risk asset and the proxy of the excess demand for stock index $\left(c_{t}\right)$ and it gives some suggestions about the possible analytical expression to link these two variables. The central part of the graph (i.e., $-3 \leq c_{t} \leq 3$ ) is almost flat, but at the two sides (i.e., $c_{t}<-3, c_{t}>3$ ) the graph shows a positive dependency.

A possible empirical model describing the previous relationship can be the following polynomial function:

$$
\widetilde{r}_{b, t}=a c_{t-1}^{3}-b c_{t-1}+d+\varepsilon_{b, t},
$$

where $\widetilde{r}_{b, t}$ are the daily returns observed for the Treasury bond and $a>0, b$ and $d$ are shape parameters to be estimated through a regression analysis which minimizes the error term. These estimates are shown in Table 1 along with their significance test. Parameter $a$, which is relevant to characterize the polynomial model, appears significant at $1 \%$ confidence. Overall the regression has an adjusted determination coefficient $\left(R^{2}\right)$ equal to .8824 and a value of the Root Mean Squared Error (RMSE) equal to .000341 .
TABLE 1: Results of the regression analysis on the daily return observed: parameter estimates, standard error of the estimates, Student's $t$ and probability of rejection.

\begin{tabular}{lcccc}
\hline Parameter & Estimate & Std. error & $t$ & $P(t \neq 0)$ \\
\hline$a$ & $9.138 E-6$ & $2.727 E-6$ & 3.35 & 0.0154 \\
$b$ & -0.00005 & 0.000076 & -0.72 & 0.5008 \\
$d$ & 0.000286 & 0.000114 & 2.51 & 0.0457 \\
\hline
\end{tabular}

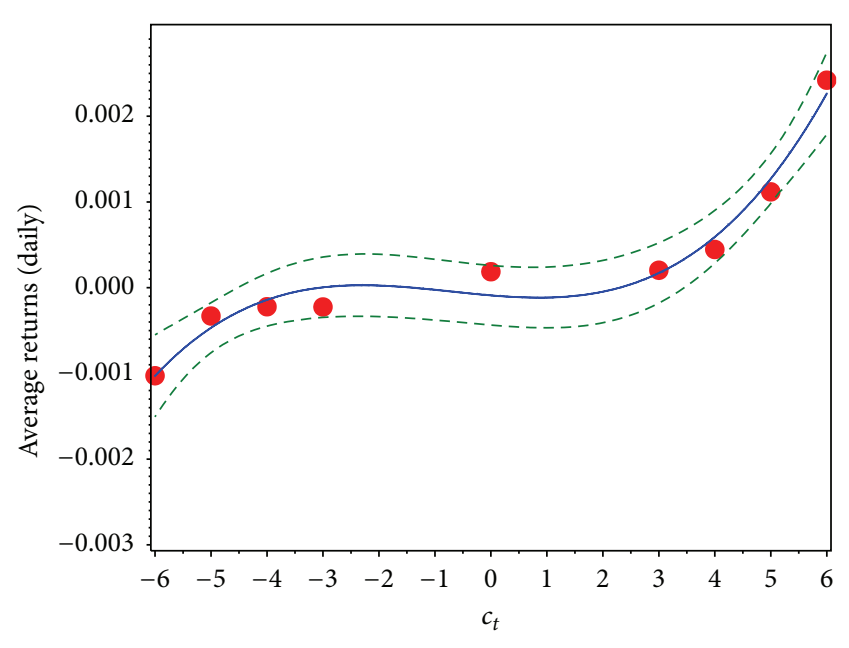

FIGURE 2: Relationship between the proxy variable of excess demand $\left(c_{t}\right)$ and the average daily returns on the US Treasury 30 years' bonds (red dots: observation period 1993-2008). $c_{t}$ is calculated on data of an NYSE stock index.

The continuous line in Figure 2 represents the expression of $\widehat{r}_{b, t}$ in system (6), where the values of the parameters $a$ and $b$ are fixed as in Table 1. What Figure 2 shows is that the two markets (i.e., stocks and bonds) strengthen their link when a clear "sentiment" in the stock market persists. Indeed, when the returns of the NYSE have all the same sign during a week, the returns on the long-term bond move substantially away from zero and show the same sign as well.

\section{Transition Dynamics}

The analysis of the model dynamics can be developed by simulating the behavior of system (6) starting from different initial values of the excess demand. We consider here two levels for the initial excess demand: large and close to zero. We additionally combine these two cases with two different market configurations: one where rational investors prevail and the other where irrational investors (of the contrarian kind) prevail. Overall we therefore analyse four scenarios.

To check the internal consistency of the model we compare two types of correlations, which we call here exante and ex-post. We refer to the ex-ante correlation as the correlation estimated by rational agents through (4) and call ex-post correlation that resulting from the simulation of model (6). Of course, for the model to be consistent, the two types of correlations should take similar values under 
TABLE 2: Different types of returns.

\begin{tabular}{lcccc}
\hline Assets & Model returns & $\begin{array}{c}\text { Expected value of returns } \\
\text { for rational agents }\end{array}$ & Empirical returns & Simulated returns \\
\hline High-risk & $\Delta p_{t}$ & $r_{s, t}$ & $\widetilde{r}_{s, t}$ & $r_{s, t}^{\varepsilon}$ \\
Low-risk & $\widehat{r}_{b, t}$ & $r_{b}$ & $\widetilde{r}_{b, t}$ & $r_{b, t}^{\varepsilon}$ \\
\hline
\end{tabular}

TABLE 3: Correlation between the returns of high-risk and low-risk assets estimated by rational agents (ex-ante correlation).

\begin{tabular}{lcc}
\hline \multirow{2}{*}{$\begin{array}{l}\text { Initial excess demand } \\
\end{array}$} & $\begin{array}{c}\text { Prevalence in the market } \\
\chi_{2}=30, \chi_{1}=0.5\end{array}$ & $\chi_{2}=3, \chi_{1}=-4$ \\
\hline Largely high or low & 0.998 & 0.112 \\
Close to zero & 0.000 & 0.113 \\
\hline
\end{tabular}

the same conditions. To obtain the estimate of the ex-post correlation, we simulate the returns of the two assets (i.e., $r_{s, t}^{\varepsilon}$ and $r_{b, t}^{\varepsilon}$ ). These returns have been obtained adding a random disturbance to $\Delta p_{t}$ and to $\widehat{r}_{b, t}$, respectively, as described in the following equations:

$$
\begin{aligned}
& r_{s, t}^{\varepsilon}=\Delta p_{t}+e_{s, t}, \\
& r_{b, t}^{\varepsilon}=\widehat{r}_{b, t}+e_{b, t},
\end{aligned}
$$

where $e_{s, t}$ and $e_{b, t}$ are i.i.d. disturbances distributed with zero expected value and standard deviations, respectively, equal to 0.02 and 0.00002 . Table 2 summarizes the different types of returns used. The purpose of adding a random disturbance is that of reducing the effect of purely deterministic simulation, where correlation between the two variables would be either 1 or -1 .

The ex-post correlation on the returns simulated by model (6) is then calculated with the standard formula:

$$
\frac{\operatorname{cov}\left(r_{s, t}^{\varepsilon}, r_{b, t}^{\varepsilon}\right)}{\sigma\left(r_{s, t}^{\varepsilon}\right) \sigma\left(r_{b, t}^{\varepsilon}\right)}
$$

where $\operatorname{cov}(\cdot, \cdot)$ and $\sigma(\cdot)$ are, respectively, the usual covariance and standard deviation functions.

Tables 3 and 4 show the values of the correlation (resp., ex-ante and ex-post) between the returns of the two assets in the four scenarios.

Comparing the two Tables we can conclude that expost and ex-ante correlations change accordingly. Observing Table 3, the large value of the ex-ante correlation occurs when rational agents dominate the market and the level of the excess demand gets very large. As already discussed, under these settings, rational agents estimate that the correlation between the traded assets (based on (4)) will run to unity. On the contrary in all the other combinations the ex-ante correlation estimate keeps at zero or slightly higher. Similar conclusions obtain observing Table 4 .
TABLE 4: Correlation between the returns of high- and lowrisk assets resulting from the simulation of the model (ex-post correlation).

$$
\text { Prevalence in the market }
$$

Initial excess demand Rational investors Contrarian speculators

\begin{tabular}{lcc} 
& $\chi_{2}=30, \chi_{1}=0.5$ & $\chi_{2}=3, \chi_{1}=-4$ \\
\hline Largely high or low & 0.971 & 0.001 \\
Close to zero & 0.064 & 0.207 \\
\hline
\end{tabular}

Figure 3 shows simulated trajectories of $r_{s, t}^{\varepsilon}$ (light color) and $r_{b, t}^{\varepsilon}$ (dark color). The cases where the initial excess demand is large are in the first row (panels (a) and (b)) and those where the initial excess demand is close to 0 are in the second row (panels (c) and (d)). Those trajectories have been obtained under the same setting that have been used for the estimate of Table 4.

The visual inspection of Figure 3 confirms the results shown in Table 4 . However the same figure also suggests to us something about the dynamics at the origin of the ex-post correlation. It is known that the theoretical model in (3) has a stable equilibrium in $w_{t}=0$ and $r_{s, t}=0$. Indeed observing the second row of Figure 3 when the initial excess demand is sufficiently close to zero, the returns show a low correlation both when rational agents prevail and when speculators prevail. Things change significantly if the initial excess demand is very large and rational agents dominate, which is the case analyzed in panel (a). It is clear in this case that the system enters into an orbital dynamic, where the returns of both assets oscillate in a synchronous way. Indeed large positive values of the excess demand sustain large returns of both the risky and the low-risk assets, following, respectively, (2) and (9).

The model explains why both returns revert severely and turn to negative. When the price of the risky asset is high enough and combines with large excess demand, rational agents change deeply their portfolio as a consequence of two circumstances:

(i) mean reversion of prices generates highly negative expected returns (see (1));

(ii) large excess demand generates high correlation estimate, which in turn reduces the opportunity to form "balanced" portfolio, as it turns out from equation (A.2) in the Appendix which determines the portfolio weight of the risky asset as a function of the 


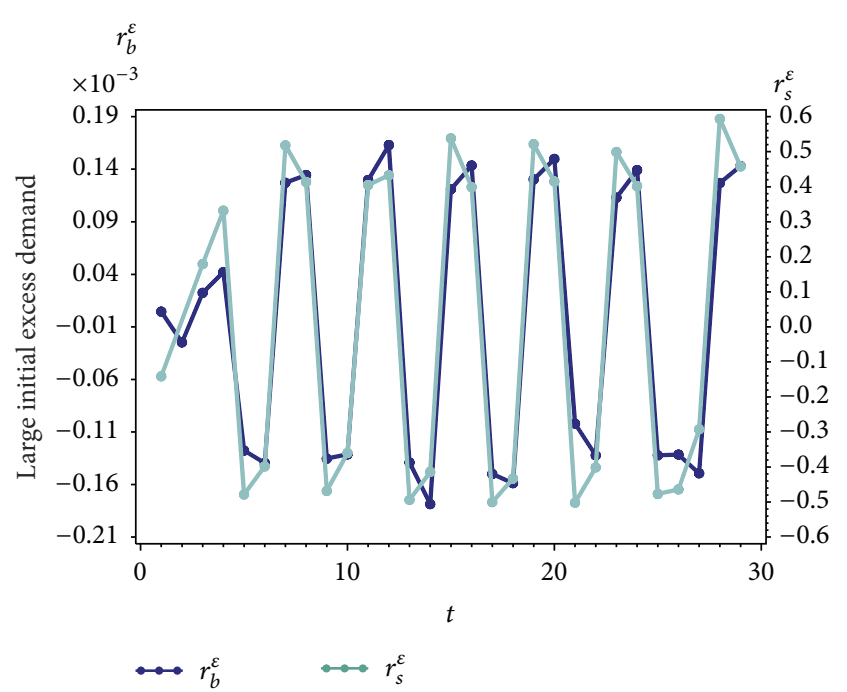

(a) $w=3, \chi_{1}=0.5$, and $\chi_{2}=30$

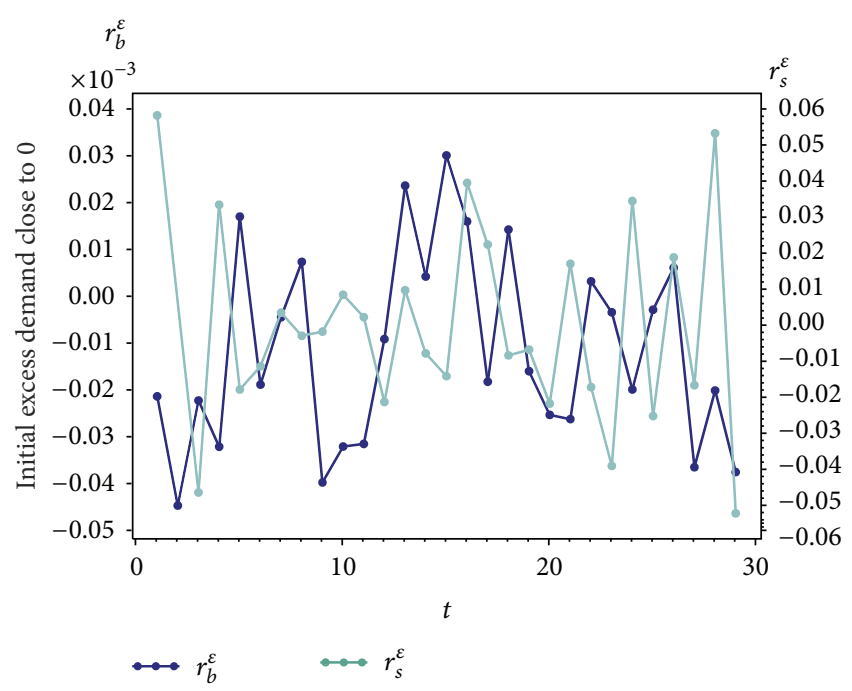

(c) $w=0.05, \chi_{1}=0.5$, and $\chi_{2}=30$

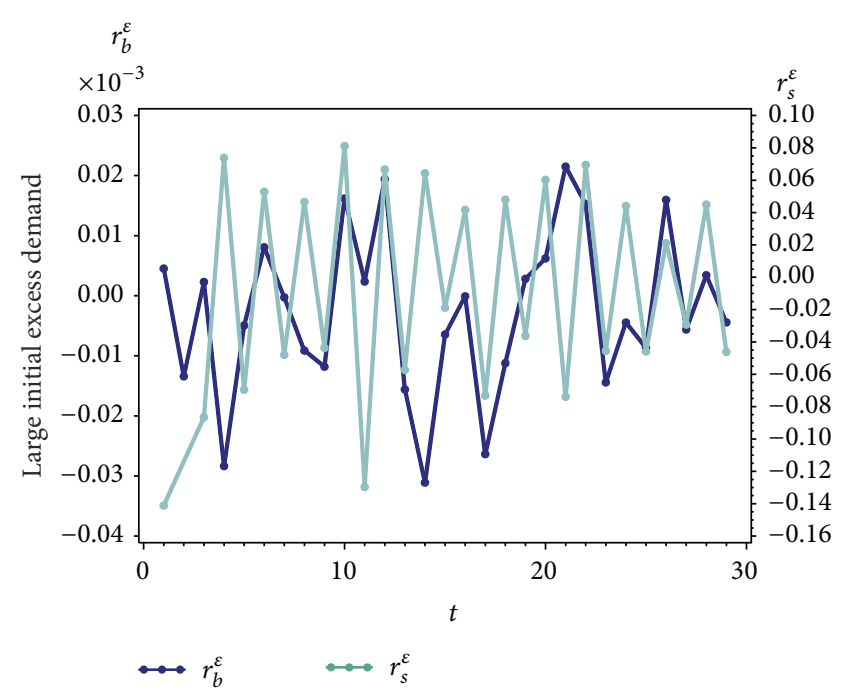

(b) $w=3, \chi_{1}=-4$, and $\chi_{2}=3$

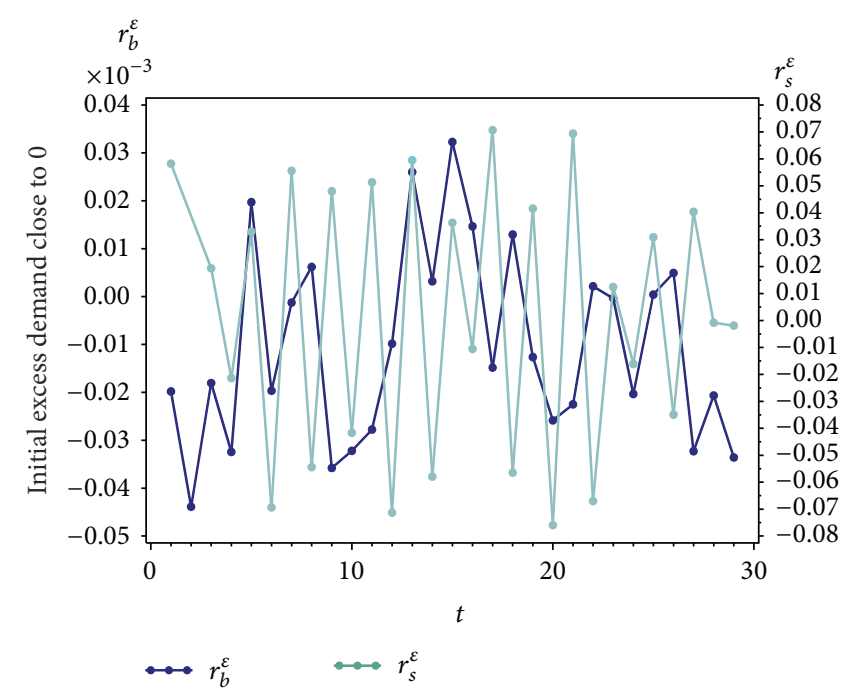

(d) $w=0.05, \chi_{1}=-4$, and $\chi_{2}=3$

FiguRE 3: Simulated trajectories of the risky and the low-risk assets. For all plots parameter values are $\alpha_{1}=0.02, \alpha_{2}=0.06, \lambda=0.05, Y=0.5$, $r_{b}=0, \bar{P}=1, k=0.20$, and $\mu=0,15$. (a) Prevalence of rational investors $\left(\chi_{2}=30\right)$ and lower importance of momentum speculators $\left(\chi_{1}=0.5\right)$; (b) prevalence of contrarian speculators $\left(\chi_{1}=-4\right)$ and lower importance of rational investors $\left(\chi_{2}=3\right)$. (c) and (d) Showing the same market composition as in (a) and (b), but the starting value of the simulation is $w=0.05$.

excess demand. Besides, extremely imbalanced portfolios are the expected result following the standard Markowitz theory when correlation between the asset values tends to 1 .

Under such conditions, rational agents put on the market large order for selling the risky asset, and if they represent the large majority of the market a large negative excess demand appears on the market. Both the risky and the low-risk asset returns are then driven down and now the story can repeat symmetrically. If on the contrary rational agents do not dominate the market as in panels (b) and (d) of Figure 3, then their weight is not enough to condition the market and the orbit does not develop.

The core of the model lies in that rational investors anticipate the correlation breakdown of the asset returns. When they do, they react directing (almost entirely) their demand towards one of the two assets and getting rid of the other. Rational agents therefore successfully anticipate the correlation breakdown between the assets, but doing so they also destroy the portfolio diversification usually protecting their investment during standard market periods. The model predicts also that when the market is sufficiently balanced with contrarian speculators, the (ex-post) correlation coefficient 
remains at low levels, because they counterbalance the procyclical behavior of rational investors.

\section{Conclusions}

The major point of this work has consisted of pushing forward the analysis of the model proposed by Falbo and Grassi [15]. Results have shown that the model is consistent with respect to the hypothesis that rational agents modify correctly their estimates of the variance and the covariance of the assets returns, anticipating the market excess demand. The relevance of this finding is in turn that of showing that putting on the shoulders of speculators all the responsibility for generating the financial crisis or the speculative bubbles, which occasionally occur on the financial markets, is not entirely justified. Indeed also risk averse, informed, rational investors can trigger such phenomena if they simply believe that large market consensus (such as large values of the excess demand) has an impact on the forthcoming correlation between the financial assets.

In extremely simplified terms this analysis has shown that also rational investors can be victim of a self-fulfilling prophecy, as soon as they attempt to anticipate what the market is going to do, which is usually attributed to speculators. Attributing to only speculators the origin of financial disasters is therefore not a correct way to understand such kind of events. Paradoxically we show that contrarian speculators act on the market as a countercyclical factor. The attitude of taking a given position on the market as a consequence of the fact that the large majority is doing the same appears rather as the triggering factor. No superior information, no diversification, and no risk averse attitude seem to supply a sufficient antidote against it.

We assumed a model for the returns of the low-risky asset, whose analytical properties capture the fundamental features observable from the financial data. This is relevant because it adds an empirical consistency to the model, next to the theoretical one discussed above.

\section{Appendix}

We shortly remind the solution of the portfolio optimization for rational agents. We refer to Falbo and Grassi [15] for a detailed explanation of computation.

The optimal quantity $q_{t}^{*}$ is obtained by searching the maximum of the following performance indicator:

$$
g=\frac{\mathbf{q}_{t}^{T} \mathbf{r}}{\mathbf{q}_{t}^{T} V_{t-1} \mathbf{q}_{t}}
$$

where $\mathbf{r}^{T}=\left[\begin{array}{ll}r_{s, t-1} & r_{b}\end{array}\right]$ is the vector of expected returns of the higher and low-risk assets and $\mathbf{q}_{t}^{T}=\left[\begin{array}{ll}q_{t} & 1-q_{t}\end{array}\right]$ is the vector of their portfolio weights.

The variance-covariance matrix $V_{t-1}$ is estimated as function of $w_{t-1}$ referring to (4). $g$ is equivalent to the Sharpe ratio, so that the optimal portfolio $\mathbf{q}_{t}^{*}=\arg \max g$ is also Markowitz efficient. The solution of the portfolio optimization problem, depending on the dynamic variables $r_{s}$ and $w$, is the following:

$$
\begin{aligned}
q_{t}^{*} & \left(w_{t-1}, r_{s, t-1}\right) \\
& = \begin{cases}-\frac{r_{b}}{r_{s, t-1}-r_{b}}+\frac{\sqrt{\left(\alpha_{1}^{2}+\alpha_{2}^{2}+2 \alpha_{1} \alpha_{2}\left(e^{\left.-\mu w_{t-1}^{2}-1\right)}\right)\left(\left(r_{s, t-1} \alpha_{2}-r_{b} \alpha_{1}\right)^{2}+2 \alpha_{1} \alpha_{2} r_{b} r_{s, t-1} e^{\left.-\mu w_{t-1}^{2}\right)}\right.\right.}}{\left(r_{s, t-1}-r_{b}\right)\left(\alpha_{1}^{2}+\alpha_{2}^{2}-2 \alpha_{1} \alpha_{2}\left(-e^{\left.-\mu w_{t-1}^{2}+1\right)}\right)\right.} & \text { if } r_{s, t-1} \neq r_{b} \\
\frac{\alpha_{1} \alpha_{2}\left(-e^{-\mu w_{t-1}^{2}}+1\right)-\alpha_{2}^{2}}{2 \alpha_{1} \alpha_{2}\left(-e^{-\mu w_{t-1}^{2}}+1\right)-\alpha_{1}^{2}-\alpha_{2}^{2}} & \text { if } r_{s, t-1}=r_{b} .\end{cases}
\end{aligned}
$$

From (A.2), $q_{t}^{*}\left(0, r_{s, t-1}\right)$ can be easily obtained as

$$
q_{t}^{*}\left(0, r_{s, t-1}\right)=-\frac{r_{b}}{r_{s, t-1}-r_{b}}+\frac{\sqrt{r_{s, t-1}^{2} \alpha_{2}^{2}+r_{b}^{2} \alpha_{1}^{2}}}{\left(r_{s, t-1}-r_{b}\right) \sqrt{\alpha_{1}^{2}+\alpha_{2}^{2}}}
$$

\section{Conflict of Interests}

The authors have no conflict of interests related to publishing this paper.

\section{References}

[1] E. Bertero and C. Mayer, "Structure and performance: global interdependence of stock markets around the crash of October
1987," European Economic Review, vol. 34, no. 6, pp. 1150-1180, 1990.

[2] M. A. King and S. Wadhwani, "Transmission of volatility between stock markets," Review of Financial Studies, vol. 3, pp. $5-33,1990$

[3] S. Calvo and C. Reinhart, "Capital flows to latin america: is there evidence of contagion effects?" in Private Capital Flows to Emerging Markets After the Mexican Crisis, G. A. Calvo, M. Goldstein, and E. Hochreiter, Eds., Institute for International Economics, Washington, DC, USA, 1996.

[4] T. Baig and I. Goldfajn, "Financial market contagion in the Asian crisis," IMF Staff Papers, vol. 46, no. 2, pp. 167-195, 1999.

[5] F. M. Longin, "The asymptotic distribution of extreme stock market returns," Journal of Business, vol. 63, pp. 383-408, 1996. 
[6] P. Hartmann, S. Straetmans, and C. G. de Vries, "Asset market linkages in crisis periods," The Review of Economics and Statistics, vol. 86, no. 1, pp. 313-326, 2004.

[7] K.-H. Bae, G. A. Karolyi, and R. M. Stulz, "A new approach to measuring financial contagion," Review of Financial Studies, vol. 16, no. 3, pp. 717-763, 2003.

[8] L. Ramchand and R. Susmel, "Volatility and cross correlation across major stock markets," Journal of Empirical Finance, vol. 5, no. 4, pp. 397-416, 1998.

[9] A. Ang and G. Bekaert, "Regime switches in interest rates," Journal of Business \& Economic Statistics, vol. 20, no. 2, pp. 163$182,2002$.

[10] F. Chesnay and E. Jondeau, "Does correlation between stock returns really increase during turbulent periods?" Economic Notes, vol. 30, no. 1, pp. 53-80, 2001.

[11] M. Loretan and W. B. English, "Evaluating correlation breakdowns during periods of market volatility," WP 658, Board of Governors of the Federal Reserve System International Finance, 2000.

[12] G. A. Karolyi and R. M. Stulz, "Why do markets move together? An investigation of U.S.-Japan stock return comovements," The Journal of Finance, vol. 51, no. 3, pp. 951-986, 1996.

[13] B. H. Boyer, "Pitfalls in tests for changes in correlations," International Finance Discussion Paper, Board of Governors of the Federal Reserve System, 1997.

[14] R. Rigobon, "Contagion: how to measure it?" in Preventing Currency Crises in Emerging Markets, S. Edwards and J. Frankel, Eds., pp. 269-334, The University of Chicago Press, Chicago, Ill, USA, 2001.

[15] P. Falbo and R. Grassi, "Market dynamics when agents anticipate correlation breakdown," Discrete Dynamics in Nature and Society, vol. 2011, Article ID 959847, 33 pages, 2011. 


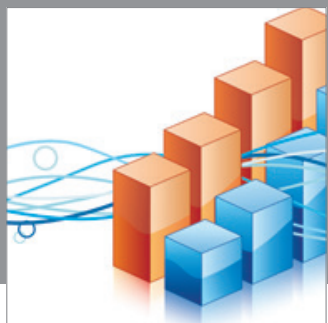

Advances in

Operations Research

mansans

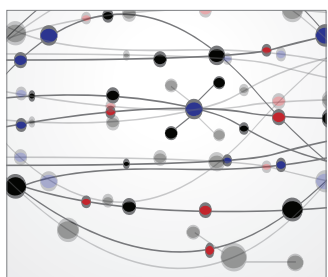

The Scientific World Journal
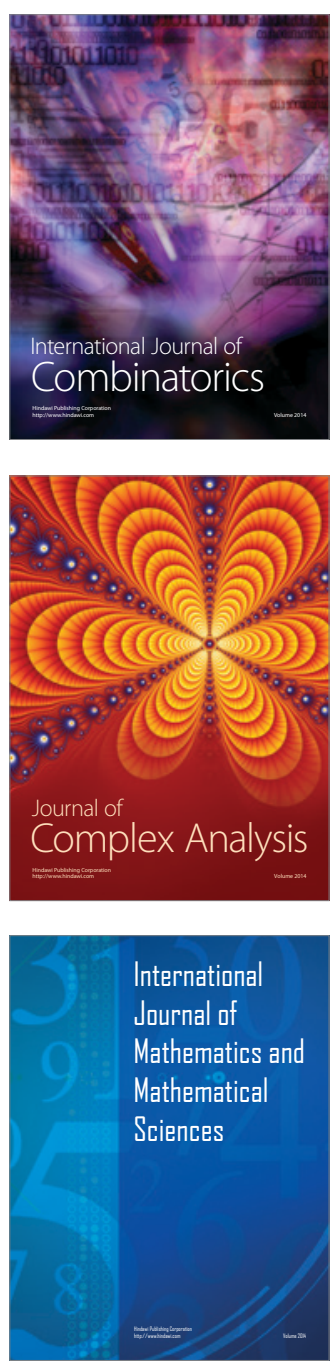
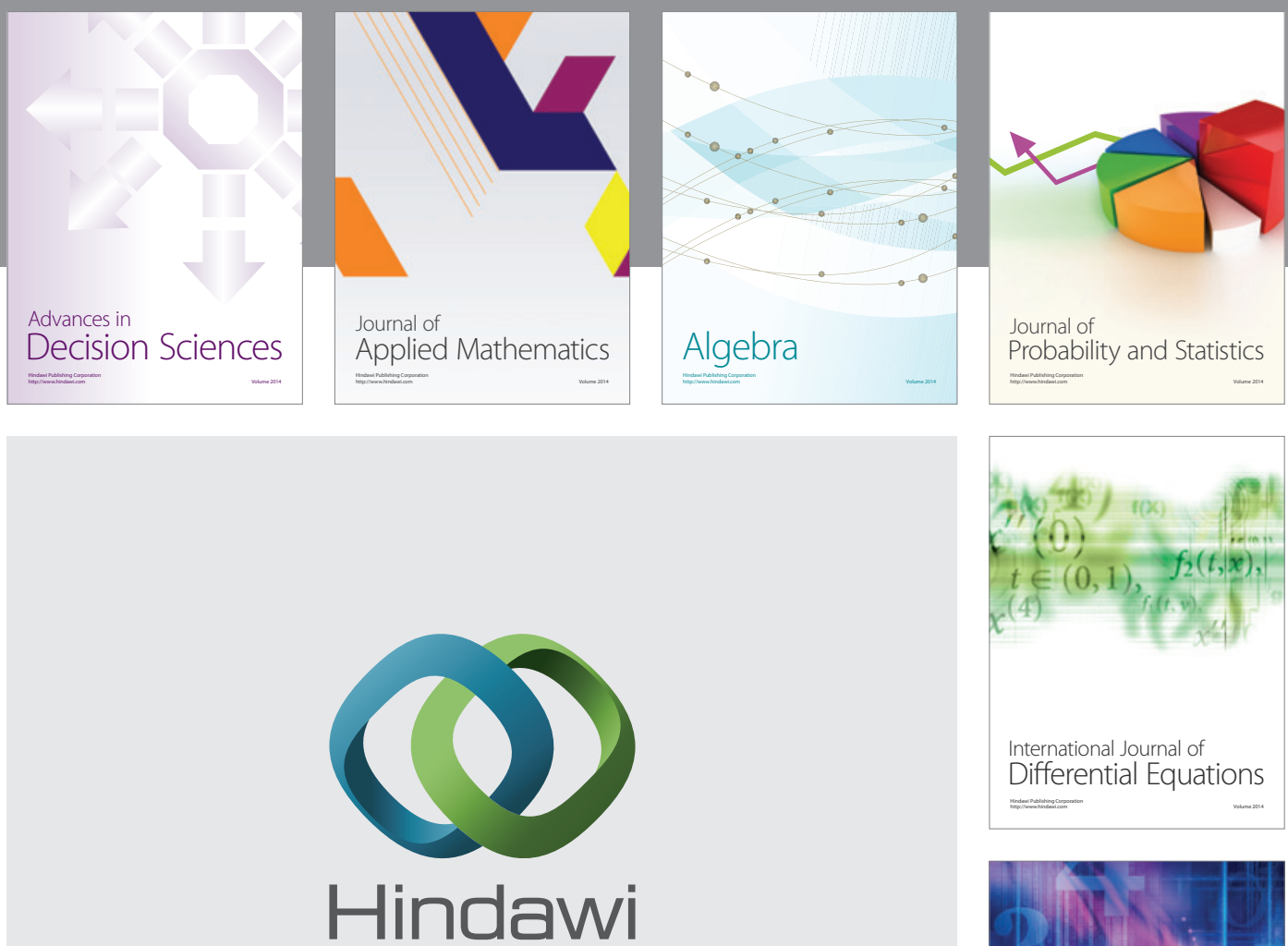

Submit your manuscripts at http://www.hindawi.com
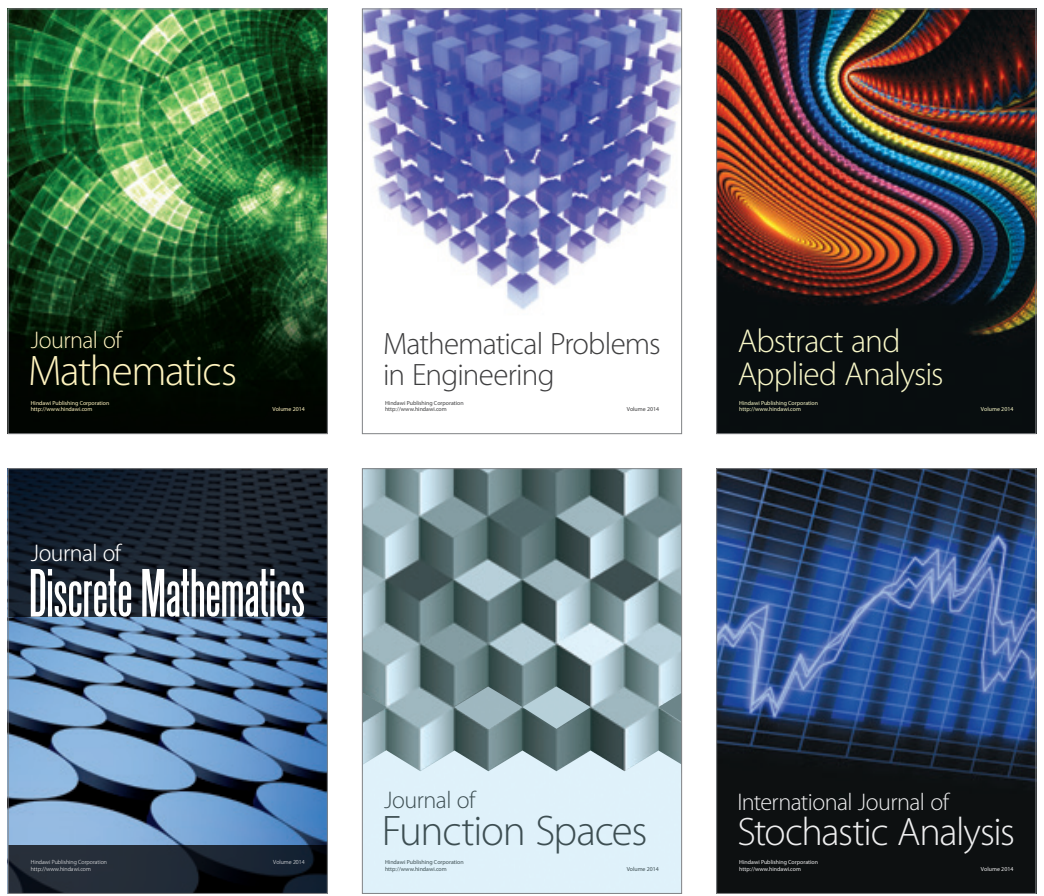

Journal of

Function Spaces

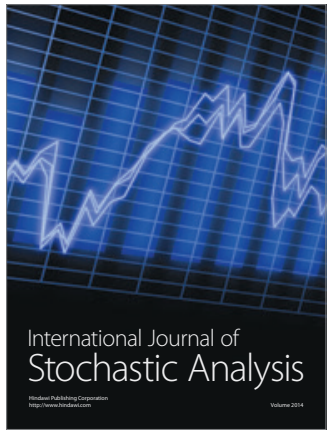

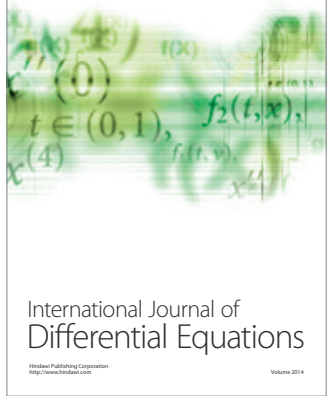
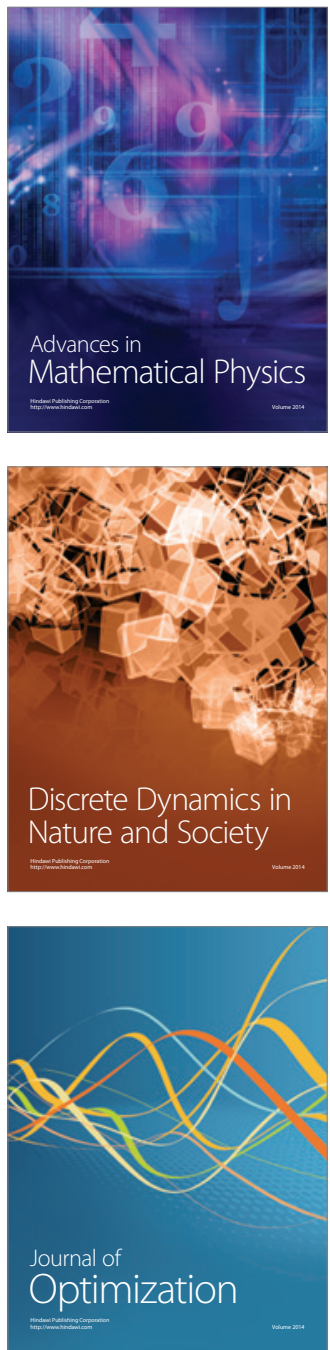